PROCEEDINGS OF THE

AMERICAN MATHEMATICAL SOCIETY

Volume 129, Number 1, Pages 311-314

S 0002-9939(00)05522-2

Article electronically published on July 27, 2000

\title{
THE SORGENFREY LINE HAS A LOCALLY PATHWISE CONNECTED CONNECTIFICATION
}

\author{
ALESSANDRO FEDELI AND ATTILIO LE DONNE
}

(Communicated by Alan Dow)

\begin{abstract}
We answer a question of Alas, Tkačenko, Tkachuk and Wilson by constructing a connected locally pathwise connected Hausdorff space in which the Sorgenfrey line can be densely embedded.
\end{abstract}

A connectification of a $T_{2}$-space $X$ is a connected Hausdorff space $Y$ in which $X$ can be densely embedded. In such a case $Y$ is called a connectification of $X$, and $X$ is said to be connectifiable. In 1977 Emeryk and Kulpa showed, in response to a question of van Douwen, that the Sorgenfrey line cannot be densely embedded in a connected $T_{3}$-space, although it is connectifiable (see [2]). In a recent paper Alas, Tkačenko, Tkachuk and Wilson asked if the Sorgenfrey line has a locally connected connectification ([1]).

The aim of this paper is to give a strongly positive answer to the above question.

We refer the reader to [3] for notations and terminology not explicitly given.

Recall that a collection of pairwise disjoint non-empty open subsets of a space $X$ is called a cellular family.

Theorem 1. The Sorgenfrey line has a locally pathwise connected connectification.

Proof. Let $S=(\mathbb{R}, \tau)$ be the Sorgenfrey line and let $D=\left\{d_{n}: n \in \mathbb{N}\right\}$ be a countable dense subset of $S$, and for every $n, i \in \mathbb{N}$ set $B(n, i)=\left[d_{n}, d_{n}+\frac{1}{i}\right.$ [ and $C(n, i)=\left[d_{n}+\frac{1}{i+1}, d_{n}+\frac{1}{i}[\right.$.

Let $\Omega$ be the subset of $\mathbb{N}^{3}$ consisting of all $\omega=(n, m, i)$ with $n<m$ and $B(n, i) \cap B(m, i)=\emptyset$. Set $\Lambda=\Omega \times(\mathbb{Z} \backslash\{0\})$.

By induction we can choose, for every $\lambda=(n, m, i, k) \in \Lambda$, a free open filter $\mathcal{F}_{\lambda}$ on $S$ with a countable base such that:

i) if $k>0$, then $C(n, i+k-1) \in \mathcal{F}_{\lambda}$;

ii) if $k<0$, then $C(m, i-k-1) \in \mathcal{F}_{\lambda}$;

iii) the family $\Phi=\left\{\mathcal{F}_{\lambda}: \lambda \in \Lambda\right\}$ is Hausdorff separated (i.e., if $\lambda \neq \lambda^{\prime}$, then there are $F \in \mathcal{F}_{\lambda}$ and $F^{\prime} \in \mathcal{F}_{\lambda^{\prime}}$ such that $F \cap F^{\prime}=\emptyset$ ).

Note that $B(n, i) \cup B(m, i) \in \mathcal{F}_{\lambda}$.

Now let $H=] 0,1\left[\cap Q \backslash\left(\left\{\frac{1}{n}: n \in \mathbb{N}\right\} \cup\left\{1-\frac{1}{n}: n \in \mathbb{N}\right\}\right)\right.$, and set $\Gamma=\Omega \times H$.

Received by the editors July 24, 1997 and, in revised form, March 25, 1999.

2000 Mathematics Subject Classification. Primary 54D35, 54D05.

Key words and phrases. Connected, Sorgenfrey line.

(C)2000 American Mathematical Society 
For each $q \in H$ set

$$
k_{q}= \begin{cases}n-1, & \text { if } \quad \frac{1}{n+1}<q<\frac{1}{n} \leq \frac{1}{2} \\ 1-n, & \text { if } \quad \frac{1}{2} \leq 1-\frac{1}{n}<q<1-\frac{1}{n+1} .\end{cases}
$$

For each $\gamma=(\omega, q) \in \Gamma$ let $\lambda_{\gamma}=\left(\omega, k_{q}\right) \in \Lambda$. Clearly, for every $\lambda \in \Lambda$, there are countably many $\gamma \in \Gamma$ such that $\lambda_{\gamma}=\lambda$.

Now for every $\gamma \in \Gamma$ choose a countably generated open filter $\mathcal{G}_{\gamma}$ on $S$ finer than $\mathcal{F}_{\lambda_{\gamma}}$ so that, for every $\lambda \in \Lambda$, the family $\left\{\mathcal{G}_{\gamma}: \gamma \in \Gamma, \lambda_{\gamma}=\lambda\right\}$ is totally Hausdorff separated, i.e., for every $\gamma$ such that $\lambda_{\gamma}=\lambda$, there is a $A_{\gamma} \in \mathcal{G}_{\gamma}$ such that $\left\{A_{\gamma}: \gamma \in \Gamma, \lambda_{\gamma}=\lambda\right\}$ is a cellular family (an easy proof of the existence of the family $\left\{\mathcal{G}_{\gamma}: \gamma \in \Gamma, \lambda_{\gamma}=\lambda\right\}$ can be found in [4]).

Set $Y=\Omega \times] 0,1[$, and for every $y=(\omega, r) \in Y$ and any $\epsilon>0$, let us denote by ]$y-\epsilon, y+\epsilon[$ the set $\{(\omega, s):|s-r|<\epsilon, s \in] 0,1[\}$.

Moreover $[y-\epsilon, y+\epsilon]$ will denote the set $\{(\omega, s):|s-r| \leq \epsilon, s \in] 0,1[\}$.

Put $\mathbb{T}=\mathbb{R} \cup Y$ and let $\tau^{*}$ be the topology on $\mathbb{T}$ generated by the base $\left\{A^{*}: A \in\right.$ $\tau$ \}, where $A^{*}$ is the subset of $\mathbb{T}$ characterized by the following properties:

1) $A^{*} \cap \mathbb{R}=A$

2) for every $y \in Y, y \in A^{*} \Leftrightarrow\left(\exists \epsilon>0: \gamma \in[y-\epsilon, y+\epsilon] \cap \Gamma \Rightarrow A \in \mathcal{G}_{\gamma}\right)$.

Observe that for every $y \in A^{*} \cap Y$ there is some $\epsilon>0$ such that $[y-\epsilon, y+\epsilon] \subset A^{*}$.

We claim that $\mathbb{T}$, endowed with the topology $\tau^{*}$, is a connected locally pathwise connected (and hence pathwise connected) $T_{2}$-space in which $S$ is densely embedded.

Clearly $S$ is a dense subspace of $\mathbb{T}$.

To check that $\mathbb{T}$ is connected and locally pathwise connected it is enough to show that $A^{*}$ is pathwise connected for every $A \in \tau$.

First let us show that for every $\omega=(n, m, i) \in \Omega$ the function $f_{\omega}:[0,1] \rightarrow \mathbb{T}$ defined by

1) $f_{\omega}(0)=d_{n}, f_{\omega}(1)=d_{m}$

2) $f_{\omega}(t)=(\omega, t)$ for every $\left.t \in\right] 0,1[$ is a path.

Let $t \in] 0,1\left[, y=f_{\omega}(t)=(\omega, t)\right.$ and take a $G \in \tau$ such that $G^{*}$ is a neighbourhood of $y$ in $\mathbb{T}$. Then there is a positive $\epsilon$ such that $G \in \mathcal{G}_{\gamma}$ for every $\gamma \in[y-\epsilon, y+\epsilon] \cap \Gamma$. Hence $f_{\omega}([t-\epsilon, t+\epsilon] \cap] 0,1[)=[y-\epsilon, y+\epsilon] \subset G^{*}$. Therefore $f_{\omega}$ is continuous on ] $0,1[$.

Now let us prove that $f_{\omega}$ is continuous at 0 . Let $G \in \tau$ such that $d_{n} \in G^{*}$. Since $d_{n} \in G$, there is a $k>0$ such that $B(n, i+k-1) \subset G$. Observe that $G \in \mathcal{F}_{(\omega, h)}$ for every $h \geq k$ (note that $\mathcal{F}_{(\omega, h)} \ni C(n, i+h-1) \subset B(n, i+h-1) \subset B(n, i+k-1)$ for every $h \geq k$ ). Therefore $G \in \mathcal{G}_{(\omega, t)}$ for every $\left.t \in\right] 0, \frac{1}{1+k}[\cap H$ (in fact let $h$ be such that $t \in] \frac{1}{2+h}, \frac{1}{1+h}\left[\cap H\right.$; then $\mathcal{G}_{(\omega, t)}$ is finer than $\left.\mathcal{F}_{(\omega, h)}\right)$.

Now let $t \in] 0, \frac{1}{1+k}\left[, y=f_{\omega}(t)=(\omega, t)\right.$ and take $\epsilon>0$ such that $[t-\epsilon, t+\epsilon]$ $\subset] 0, \frac{1}{1+k}\left[\right.$ and $G \in \mathcal{G}_{\gamma}$ for every $\gamma \in[y-\epsilon, y+\epsilon] \cap \Gamma$. Then $y \in G^{*}$. Hence $f_{\omega}\left(\left[0, \frac{1}{1+k}[) \subset G^{*}\right.\right.$, and $f_{\omega}$ is continuous at 0 . Similarly it can be shown that $f_{\omega}$ is continuous at 1 . Therefore $f_{\omega}$ is a path.

Now let us show that for every $A \in \tau$ and $x, y \in A^{*}$ there is a path in $A^{*}$ between $x$ and $y$.

i) $x=d_{n}$ and $y=d_{m}$. Let $i$ be such that $B(n, i) \cup B(m, i) \subset A$; then $A \in \mathcal{G}_{\gamma}$ for every $\gamma=(\omega, q) \in \Gamma$, where $\omega=(n, m, i)$ and $n<m$. Now set $z=(\omega, t)$ with 
$t \in] 0,1\left[\right.$ and let us show that $z \in A^{*}$. Take a positive $\epsilon$; then $A \in \mathcal{G}_{\gamma}$ for every $\gamma \in[y-\epsilon, y+\epsilon] \cap \Gamma$. Hence $z \in A^{*}$. Therefore $f_{\omega}$ is a path between $x$ and $y$ in $A^{*}$.

ii) $x=d \in A \cap D$ and $y \in A$. Let $\left\{B_{n}: n \in \mathbb{N}\right\}$ be a decreasing base for $S$ in $y$ such that $B_{1}=A, C_{n}=B_{n} \backslash c l_{X}\left(B_{n+1}\right) \neq \emptyset$ and $d \in C_{1}$. Put $d_{n_{1}}=d$ and take $d_{n_{k}} \in C_{k} \cap D$ with $n_{k}<n_{k+1}$, for every $k \geq 2$. Now choose, for every $k \in \mathbb{N}$, $i_{k}$ so that $B\left(n_{k}, i_{k}\right) \cup B\left(n_{k}, i_{k-1}\right) \subset C_{k}$. Set $\omega_{k}=\left(n_{k}, n_{k+1}, i_{k}\right)$ and observe that $f_{\omega_{k}}$ is a path between $d_{n_{k}}$ and $d_{n_{k+1}}$ contained in $\left(B\left(n_{k}, i_{k}\right) \cup B\left(n_{k+1}, i_{k}\right)\right)^{*}$ (and hence in $B_{k}^{*}$ ). Since $\left\{B_{n}^{*}: n \in \mathbb{N}\right\}$ is a base for $\mathbb{T}$ at $y$ and, for every $n \in \mathbb{N}$, all but finitely many $f_{\omega_{k}}$ are contained in $B_{n}^{*}$, it is possible to find a path in $A^{*}$ between $x$ and $y$.

iii) $x=d \in A$ and $y=(\omega, t) \in A^{*} \backslash A$. Take $\epsilon>0$ such that $[y-\epsilon, y+\epsilon] \subset A^{*}$. Since $[y-\epsilon, y+\epsilon]$ is a path, we may assume that $y \in \Gamma$. If $B^{*}$ is a neighbourhood of $y$, then there is $\epsilon>0$ such that $B \in \mathcal{G}_{\gamma}$ for each $\gamma \in[y-\epsilon, y+\epsilon] \cap \Gamma$, in particular $B \in \mathcal{G}_{y}$.

Now if $\mathcal{B}$ is a countable base of $\mathcal{G}_{y}$, then $\left\{B^{*}: B \in \mathcal{B}\right\}$ is a local $\pi$-base of $\mathbb{T}$ at $y$. Arguing as in the previous case we can find a path in $A^{*}$ between $x$ and $y$.

Now it remains to show that $\mathbb{T}$ is a Hausdorff space. So let us consider two distinct points $x$ and $y$ in $\mathbb{T}$.

1) $x, y \in \mathbb{R}$. Choose $A, B \in \tau$ such that $A \cap B=\emptyset, x \in A$ and $y \in B$. Clearly $A^{*}$ and $B^{*}$ are open in $\mathbb{T}, x \in A^{*}$ and $y \in B^{*}$. We claim that $A^{*} \cap B^{*}=\emptyset$. Suppose not, and take $p \in A^{*} \cap B^{*}$. Since $p \in Y$, there is some $\epsilon>0$ such that $A, B \in \mathcal{G}_{\gamma}$ for every $\gamma \in[y-\epsilon, y+\epsilon] \cap \Gamma$, a contradiction.

2) $x \in \mathbb{R}$ and $y=(\omega, r) \in Y$. It is easy to see that there are a sufficiently small $\epsilon>0$ and $\mathcal{F}_{1}, \mathcal{F}_{2} \in \Phi$ such that, for every $\gamma \in[y-\epsilon, y+\epsilon] \cap \Gamma, \mathcal{F}_{\lambda_{\gamma}}=\mathcal{F}_{1}$ or $\mathcal{F}_{\lambda_{\gamma}}=\mathcal{F}_{2}$. Since $\mathcal{F}_{1}$ and $\mathcal{F}_{2}$ are free filters, there are $A_{1} \in \mathcal{F}_{1}, A_{2} \in \mathcal{F}_{2}$ and a neighbourhood $B$ of $x$ such that $A_{i} \cap B=\emptyset, i \in\{1,2\}$. Set $A=A_{1} \cup A_{2}$ and observe that $A^{*} \cap B^{*}=\emptyset$. Clearly $x \in B^{*}$; moreover $y \in A^{*}$ (since $A \in \mathcal{F}_{1}$ and $A \in \mathcal{F}_{2}$, it follows that $A \in \mathcal{F}_{\lambda_{\gamma}} \subset \mathcal{G}_{\gamma}$ for every $\left.\gamma \in[y-\epsilon, y+\epsilon] \cap \Gamma\right)$.

3) $x=(\omega, r), y=\left(\omega^{\prime}, r^{\prime}\right) \in Y$, with $\omega \neq \omega^{\prime}$. Take $\epsilon>0$ and $\mathcal{F}_{i} \in \Phi$ for every $i \in\{1,2,3,4\}$ so that:

i) $\mathcal{F}_{i} \neq \mathcal{F}_{j}$ whenever $i \neq j$;

ii) $\mathcal{F}_{1} \subset \mathcal{G}_{\gamma}$ or $\mathcal{F}_{2} \subset \mathcal{G}_{\gamma}$ for every $\gamma=(\omega, t) \in[x-\epsilon, x+\epsilon] \cap \Gamma$;

iii) $\mathcal{F}_{3} \subset \mathcal{G}_{\gamma}$ or $\mathcal{F}_{4} \subset \mathcal{G}_{\gamma}$ for every $\gamma=\left(\omega^{\prime}, t\right) \in[y-\epsilon, y+\epsilon] \cap \Gamma$.

Since $\left\{\mathcal{F}_{1}, \mathcal{F}_{2}, \mathcal{F}_{3}, \mathcal{F}_{4}\right\}$ is Hausdorff separated, we can choose $A_{i} \in \mathcal{F}_{i}$ for every $i \in\{1,2,3,4\}$ in such a way that the family $\left\{A_{1}, A_{2}, A_{3}, A_{4}\right\}$ is cellular.

Put $A=A_{1} \cup A_{2}, B=A_{3} \cup A_{4}$. It is clear that $A^{*}$ and $B^{*}$ are open sets of $\mathbb{T}$ such that $x \in A^{*}$ and $y \in B^{*}$. Since $A \cap B=\emptyset$, it follows that $A^{*}$ and $B^{*}$ are disjoint.

4) $y_{1}=\left(\omega, r_{1}\right), y_{2}=\left(\omega, r_{2}\right) \in Y$, with $r_{1}<r_{2}$.

If there is some $n \geq 2$ such that $\left.\frac{1}{n} \in\right] r_{1}, r_{2}\left[\right.$ or $\left.1-\frac{1}{n} \in\right] r_{1}, r_{2}[$, then there are $\epsilon>0$ and $\mathcal{F}_{1}, \mathcal{F}_{2}, \mathcal{F}_{3}, \mathcal{F}_{4}$ as in the previous case. So let us suppose that there is $n \geq 1$ such that $\frac{1}{n+1} \leq r_{1}<r_{2} \leq \frac{1}{n}$ (the case $1-\frac{1}{n} \leq r_{1}<r_{2} \leq 1-\frac{1}{n-1}$ is similar). Then there are $\epsilon>0$ and $\mathcal{F}, \mathcal{F}_{1}, \mathcal{F}_{2} \in \Phi$ such that

i) $\left[y_{1}-\epsilon, y_{1}+\epsilon\right] \cap\left[y_{2}-\epsilon, y_{2}+\epsilon\right]=\emptyset$;

ii) $\mathcal{F} \subset \mathcal{G}_{\gamma}$ or $\mathcal{F}_{1} \subset \mathcal{G}_{\gamma}$ for every $\gamma \in\left[y_{1}-\epsilon, y_{1}+\epsilon\right] \cap \Gamma$.

iii) $\mathcal{F} \subset \mathcal{G}_{\gamma}$ or $\mathcal{F}_{2} \subset \mathcal{G}_{\gamma}$ for every $\gamma \in\left[y_{2}-\epsilon, y_{2}+\epsilon\right] \cap \Gamma$.

Choose $F \in \mathcal{F}, F_{1} \in \mathcal{F}_{1}$ and $F_{2} \in \mathcal{F}_{2}$ so that the family $\left\{F, F_{1}, F_{2}\right\}$ is cellular.

Since the family $\left\{\mathcal{G}_{\gamma}: \gamma \in \Gamma, \mathcal{F} \subset \mathcal{G}_{\gamma}\right\}$ is totally Hausdorff separated, take $A_{\gamma} \in \mathcal{G}_{\gamma}$ such that $A_{\gamma} \subset F$ and the family $\left\{A_{\gamma}: \gamma \in \Gamma, \mathcal{F} \subset \mathcal{G}_{\gamma}\right\}$ is cellular.

Set $C_{i}=F_{i} \cup \bigcup\left\{A_{\gamma}: \gamma \in\left[y_{i}-\epsilon, y_{i}+\epsilon\right] \cap \Gamma, \mathcal{F} \subset G_{\gamma}\right\}$ and note that $C_{1} \cap C_{2}=\emptyset$. 
Now it is enough to observe that $C_{1}^{*}$ and $C_{2}^{*}$ are disjoint open subsets of $\mathbb{T}$ such that $y_{i} \in C_{i}^{*}, i \in\{1,2\}$.

Remark 1. A space $X$ is called feebly compact if every countable open cover of $X$ has a finite subfamily whose union is dense. Observe that if $G$ is an open subset of a space $X$ whose closure is not feebly compact, then $G$ is an element of a free open filter on $X$ with a countable base (see Lemma 3.7 in [6]). It is easy to see that in the proof of Theorem 1 we use only the fact that the Sorgenfrey line is a first countable separable Hausdorff space in which every non-empty open subset has non feebly compact closure.

Therefore we have the following more general result.

Theorem 2. Every first countable separable Hausdorff space with no non-empty open subsets with feebly compact closure has a locally pathwise connected connectification.

A space is called pathwise connectifiable if it can be densely embedded in a pathwise connected Hausdorff space (4). Since every non-empty countable Hausdorff space without isolated points is not feebly compact ( $[5$, Theorem 5.2), we have the following

Corollary 1 ([4]). Every countable first countable Hausdorff space without isolated points is pathwise connectifiable.

\section{REFERENCES}

1. O.T. Alas, M.G. Tkačenko, V.V. Tkachuk and R.G. Wilson, Connectedness and local connectedness of topological groups and extensions, preprint.

2. A. Emeryk and W. Kulpa, The Sorgenfrey line has no connected compactification, Comm. Math. Univ. Carolinae 18 (1977), 483-487. MR 57:1422

3. R. Engelking, General Topology, Sigma series in Pure Mathematics 6, Heldermann Verlag, Berlin, 1989. MR 91c:54001

4. A. Fedeli and A. Le Donne, Dense embeddings in pathwise connected spaces, Topology and its Applications 96 (1999), 15-22. CMP 99:16

5. J. Porter and J. Vermeer, Spaces with coarser minimal Hausdorff topologies, Trans. Amer. Math. Soc. 289 (1985), 59-71. MR 86h:54030

6. S. Watson and S. Wilson, Embeddings in connected spaces, Houston J. Math. 19 (1993), no. 3, 469-481. MR 94k:54040

Department of Mathematics, University of L'Aquila, 67100 L'Aquila, Italy

E-mail address: alessandro.fedeli@axscaq.aquila.infn.it

Department of Mathematics, University of Rome "La Sapienza", 00100 Rome, Italy

E-mail address: ledonne@mat.uniroma1.it 